\title{
Insights into the Exceptional Crystallographic Order of Biominerals Using Dark- Field X-ray Microscopy
} \author{
Detlefs $^{1}$ \\ 1. European Synchrotron Radiation Facility, Grenoble, France. \\ 2. Danmarks Tekniske Universitet, Institut for Fysik, Kgs. Lyngby, Denmark. \\ 3. Ocas Zwijnaarde, Zwijnaarde, Belgium. \\ * pcook@esrf.fr
}

$\underline{\text { Phil K. Cook }}^{1 *}$, Hugh Simons ${ }^{2}$, Anders C. Jakobsen ${ }^{2}$, Can Yildirim ${ }^{1,3}$, Henning F. Poulsen ${ }^{3}$, Carsten

Biominerals are hierarchical materials found throughout nature [1]. Examples are widespread, including bone, tooth, corals, shells, and otoliths. Biominerals form as a combination of inorganic mineral and organic template under strict biological control by the organism, yielding an exceptional control of final structure in both crystallography and habit [2]. This control has evolved to produce materials whose properties are optimised for particular functions, such as wear resistance for teeth or strength and crack resistance for protective shells. Like nature, materials science aims to produce function-optimised materials. Bio-inspired structures are growing increasingly common as scientists seek to understand and replicate natural growth processes [3]. Nonetheless, many aspects of biomineralization remain that are not fully understood.

The new hard X-ray microscope constructed at ESRF ID06 provides unique insight into the structure of polycrystalline materials $[4,5,6]$. Inspired by dark-field transmission electron microscopy, an X-ray compound refractive lens is placed in the Bragg diffracted beam from a selected grain. This lens focuses and magnifies the image onto a detector, producing a projection of the real-space shape of a grain. By

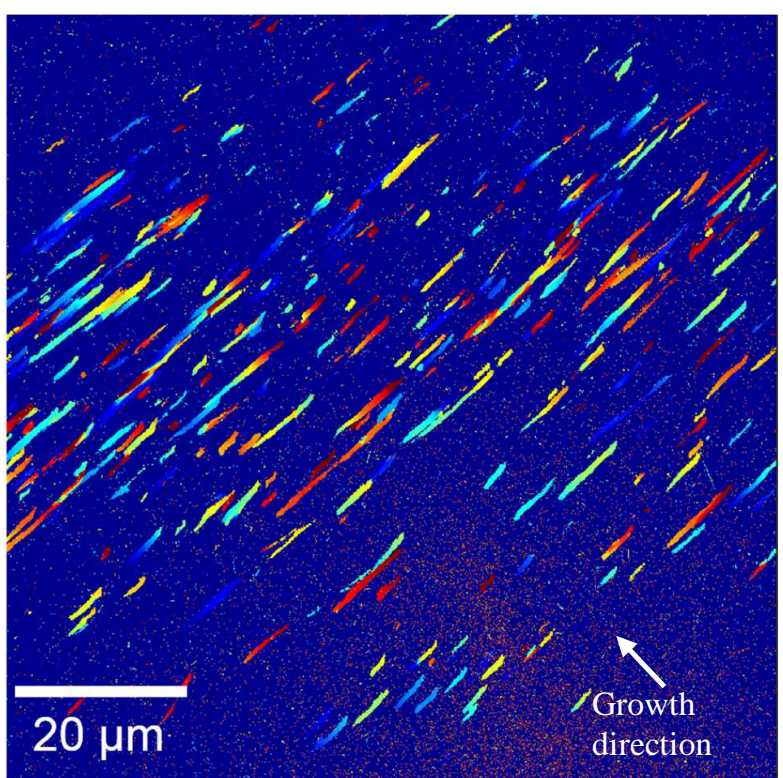

Figure 1. Orientations of nacre platelets in a mussel shell imaged by dark-field X-ray microscopy. Colour scale indicates orientation. taking a series of images at different angular positions, mosaicity and lattice strain can be determined with exceptional precision at a real-space resolution of $\sim 100 \mathrm{~nm}$. The use of X-rays permits analysis of samples up to $1 \mathrm{~mm}$ thick with little preparation necessary, making it a valuable tool for bulk-scale studies of polycrystalline materials.

In the present work, we have examined two types of biominerals using dark-field X-ray microscopy. First, we examined the nacreous layer in mussel shell. Nacre grows in a brick-and-mortar-like assembly, composed of calcium carbonate platelets surrounded by proteinaceous organic matrix. A piece of shell from the edge was broken off and mounted on the sample support. Examination with the darkfield microscope permitted visualization of a multitude of platelets with a broad range in orientation (Figure 1). Reflections were observed across the full scan range of $0.6^{\circ}$ in both base tilt and 
azimuth. This unique view of the platelets in situ illustrates their highly controlled assembly and inter-platelet relationships.

The second biomineral examined was fish otolith. Otoliths are calcium carbonate accretions that grow in the inner ear of vertebrate fish. They are composed of aragonite fibres which extend from the nucleus to the outer surface. A hierarchical structure is present, comprising the fibre, bundles of prismatic crystalline units, and nanogranules [7]. Figure 2 shows the dark field microscopy image of a $100 \mu \mathrm{m}$ long otolith fibre. Bundles of prismatic crystalline units are visible along its length. The interruptions between bundles along the growth axis likely correspond to daily growth layers governed by the individual's circadian rhythm. Single crystalline units can be seen stacked side by side across the

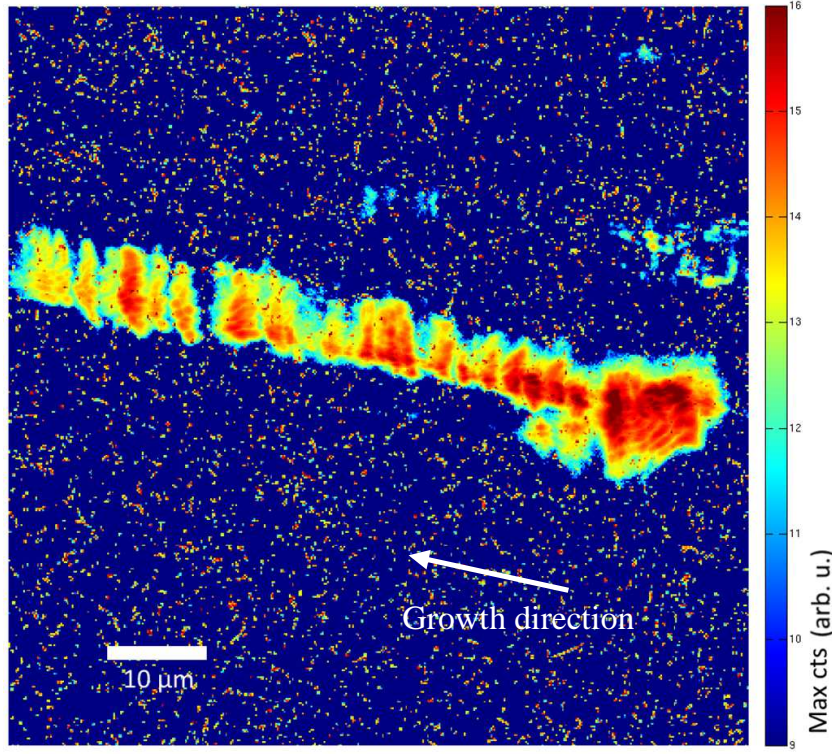

Figure 2. Aragonite fibre in an otolith imaged using dark-field X-ray microscopy.

fibre. Individual nanogranules cannot be resolved, but small clusters can be seen. The prismatic crystalline units are closely oriented with respect to the growth axis, with the crystal $c$ axes coherent to $+/-2^{\circ}$. The orientation of the $a$ and $b$ axes, however, appears to be isotropic, with prisms occupying a wide range of orientations around the $c$ axis.

The dark-field X-ray microscope at ESRF ID06 is a powerful new tool for the characterization of polycrystalline materials. In particular, it shows promise to bring new perspectives to the study of biominerals, permitting a unique in situ view with an exceptional angular resolution, providing a useful complement to current techniques [8].

\section{References:}

[1] P. Fratzl and R. Weinkamer, Prog. Mater. Sci., 52 (2007), p. 1263.

[2] S. Weiner and P. M. Dove, Rev. Mineral. Geochem., 54 (2003), p. 1.

[3] D. Zöllner, E. Reich, and I. Zlotnikov, Cryst. Growth Des., 17 (2017), p. 5023.

[4] H. Simons et al, Nat. Comm., 6 (2015), p. 6098.

[5] H. F. Poulsen et al, J. Appl Cryst., 50 (2017), p. 1441.

[6] H. Simons et al, Microsc. Microanal., in press.

[7] Y. Dauphin and E. Dufour, Micron, 39 (2008), p. 891.

[8] The authors thank Élise Dufour (UMR 7209 CNRS/Muséum National d'Histoire Naturelle) for providing otolith samples for study. 